\title{
BMJ Open Outcomes of an individual counselling programme in Grozny, Chechnya: a randomised controlled study
}

\author{
Annick Lenglet, ${ }^{1}$ Barbara Lopes-Cardozo, ${ }^{2}$ Leslie Shanks, ${ }^{1}$ Curtis Blanton, ${ }^{2}$ \\ Concetta Feo, ${ }^{3}$ Zalina Tsatsaeva, ${ }^{3}$ Kyuri Idrisov, ${ }^{4}$ Paul A Bolton, ${ }^{5}$ Giovanni Pintaldi ${ }^{1}$
}

To cite: Lenglet A, LopesCardozo B, Shanks L, et al. Outcomes of an individual counselling programme in Grozny, Chechnya: a randomised controlled study. BMJ Open 2018;8:e019794. doi:10.1136/ bmjopen-2017-019794

- Prepublication history and additional material for this paper are available online. To view these files, please visit the journal online (http://dx.doi. org/10.1136/bmjopen-2017019794).

Received 25 September 2017

Revised 23 May 2018

Accepted 12 July 2018

\section{Check for updates}

(C) Author(s) (or their employer(s)) 2018. Re-use permitted under CC BY-NC. No commercial re-use. See rights and permissions. Published by BMJ.

${ }^{1}$ Médecins Sans Frontières, Operational Centre Amsterdam, Amsterdam, The Netherlands ${ }^{2}$ Emergency Response and Recovery Branch, Division of Global Health Protection, Center for Global Health, Centers for Disease Control and Prevention, Atlanta, Georgia, USA

${ }^{3}$ Médecins Sans Frontières, Moscow, Russia

${ }^{4}$ Psychiatry Department, Chechnya State University, Grozny, Republic of Chechnya ${ }^{5}$ Departments of International Health and Mental

Health,Bloomberg School of Public Health, Johns Hopkins University, Baltimore, Maryland, USA

\section{Correspondence to} Annick Lenglet;

Annick.Lenglet@amsterdam. msf.org

\section{ABSTRACT}

Objectives To evaluate the effectiveness of individual counselling on functioning of clients participating in a mental health intervention in a humanitarian setting. Design Randomised controlled trial.

Setting Mental health programme implemented by Médecins Sans Frontières in Grozny, Republic of Chechnya. Participants 168 eligible clients were randomly assigned to the intervention and waitlisted (2 months) arms between November 2014 and February 2015.

Intervention Individual counselling sessions.

Main outcome measures Change in functioning was measured using the Short Form 6 (SF6) and genderspecific locally adapted Chechen functioning instruments in the intervention group at the end of counselling and the waitlisted group after their waitlisted period. Unadjusted differences in gain scores (DGSs) between intervention and waitlisted groups were calculated with effect size (Cohen's d) for both tools. Linear regression compared the mean DGS in both groups.

Results The intervention group $(n=78)$ improved compared with waitlisted controls $(n=80)$ on the SF6 measures with moderate to large effect sizes: general health (DGS 12.14, $d=0.52$ ), body pain (DGS 10.26, $d=0.35$ ), social support (DGS 16.07, $d=0.69$ ) and emotional functioning (DGS 16.87, $d=0.91$ ). Similar improvement was seen using the Chechen functioning instrument score (female DGS $-0.33, d=0.55$; male DGS $-0.40, d=0.99)$. Adjusted analysis showed significant improvement $(p<0.05)$ in the intervention group for all SF6 measures and for the Chechen functioning instrument score in women but not men $(p=0.07)$.

Conclusions Individual counselling significantly improved participants' ability to function in the intervention group compared with the waitlisted group. Further research is needed to determine whether similar positive results can be shown in other settings and further exploring the impact in male clients' population.

Trial registration number NTR4689.

\section{INTRODUCTION}

Mental health (MH) needs of people affected by emergencies are undisputed. Recognition of the need for scale-up of MH programming in lower income countries and humanitarian contexts was solidified by the WHO mhGAP 2010 Intervention Guide, updated in $2016 .{ }^{1}$

\section{Strengths and limitations of this study}

Randomised controlled trial conducted in a humanitarian setting in a scientifically rigorous way.

Very low rate of loss to follow-up in the intervention and waitlisted group throughout the study period.

- Intervention impact evaluated using different psychological measurement instruments.

- Participant recruitment occurred at hospitals, thus the study population might not be fully representative of the general population in Grozny.

Blinding of study counsellors to the intervention and waitlisted group could not be ensured in all cases.

WHO recently issued Problem Management Plus (PM+) guidance to support implementation of individual psychological support for adults in communities affected by adversity. ${ }^{2}$ The guidelines recommend the implementation of brief $\mathrm{MH}$ interventions during humanitarian emergencies as often these contexts limit the ability to implement longer term counselling strategies. Furthermore, the guidelines recognise that fully trained $\mathrm{MH}$ workers in emergency-affected areas are often limited and thus MH interventions often rely on non-specialised MH staff.

MH interventions involving non-specialised counselling for adults conducted in emergency settings have shown to have a beneficial effect on post-traumatic stress disorder (PTSD) in meta-analysis conducted on seven studies in 2011. ${ }^{3}$ Several randomised controlled trials (RCTs) looking at different $\mathrm{MH}$ interventions in humanitarian settings have since been published. ${ }^{4}$ However, the interventions vary in their approach, target groups, duration of intervention and types of MH staff used. Cognitive-behavioural therapy (CBT) and cognitive processing therapy have been shown to have positive outcomes on depression, anxiety and PTSD in RCTs in Iraq and Iraqi Kurdistan in war-traumatised adults and survivors of violence, torture and 
militant attacks. ${ }^{5-7}$ Several other RCTs have addressed other methods of psychosocial support including transdiagnostic approaches, Common Elements Treatment Approach (CETA), behavioural interventions and psychosocial counselling in individual and group interventions in various countries. ${ }^{8-14}$

The main MH intervention implemented by Médecins Sans Frontières - Operational Centre Amsterdam (MSFOCA) is the provision of individual counselling. ${ }^{15}$ It aims to enhance clients' functionality, reduce symptoms of mental distress and identify new coping strategies. ${ }^{15}$ The intervention does not address severe MH disorders such as severe depression, bipolar disorder or psychosis. The counselling approach is based on principles derived from brief trauma-focused therapy and CBT's techniques integrated into the cultural context. ${ }^{16}$ A review of 18 MSF-OCA individual-focused non-specialised counselling programmes comprising 15000 clients showed positive outcomes among those returning for follow-up. ${ }^{15}$ However, no evaluation using a control group to determine the impact of this form of individual counselling was conducted before this study.

Grozny, the capital of the Republic of Chechnya, has been exposed to different waves of violence and humanitarian strife since 2001. Between 2001 and 2003, MSF provided $\mathrm{MH}$ services to internally displaced people with trauma-related symptoms resulting from heavy shelling and massive explosions. Since 2008, the situation in Chechnya has improved, but there continued to be ongoing violence and insecurity. Between 2008 and 2017, MSF provided individual counselling and psychosocial services support to the population through three hospitals in Grozny, one hospital in Shatoy district and one hospital in Vedeno district.

The primary objective of our study was to estimate the effectiveness of the individual counselling intervention in Grozny on the daily functioning of clients enrolled using an individual level stepped-wedge RCT. Functioning was chosen as the main outcome of the study as this is the primary goal in the MSF individual counselling approach. Our hypothesis was that the individual counselling would lead to improved functioning in daily life for intervention clients compared with waitlisted clients.

\section{METHODS}

\section{Study location and design}

The study was conducted in Grozny, capital of the Republic of Chechnya, and implemented in three Ministry of Health hospitals where MSF's MH programme functions. The design was an RCT using a stepped-wedge design at the individual level. ${ }^{17}$ Stepped-wedge randomised trial designs involve sequential roll-out of an intervention to participants (individuals or clusters) over a number of time periods. ${ }^{17}$ Study participants were randomly assigned to intervention or waitlisted control arms, based on order of arrival for counselling (figure 1), and thus by the end of the random allocation, all individuals enrolled in the study would have received the individual counselling intervention. The intervention group received individual counselling immediately after enrolment. The waitlisted group had their counselling deferred for 2 months. The 2-month waiting period was based on the average length of treatment for clients enrolled in the programme in Grozny before the study and on a community consultation exercise implemented prior to the study. ${ }^{18}$ The intervention group completed measurements at enrolment/ preintervention (B/T0), postintervention (T1) and 3 and 6 months postintervention (T2 and T3). The waitlisted group completed measurements at enrolment (baseline/B), preintervention immediately before the start of counselling (T0), following the end of counselling (T1) and 3 months later (T2) (figure 1). The waitlisted group was not followed up at 6 months postintervention due to practical considerations around the duration of the trial.

\section{Participants}

All clients seeking care at the MSF-OCA MH programme in Grozny between November 2014 and February 2015 were considered for eligibility. Clients were self-referred or referred by staff and volunteers at the three hospitals. Clients included outpatients but could also include those admitted to the hospitals in question. Clients were eligible for inclusion if they were aged 18 years or older, able to provide informed consent and willing and able to return for follow-up. Clients were not eligible if they had cognitive, visual or other impairments that would limit their ability to participate, were considered at acute risk of suicide, had a severe MH disorder requiring medications or had been enrolled in MSF counselling services in the previous 6 months. Inclusion and exclusion criteria were established at presentation by a study interviewer/ counsellor using a pre-established checklist.

All eligible clients were asked if they would consent to undergoing screening using the Hopkins Symptom Checklist-25 (HSCL-25). All those scoring 1.75 or greater on this questionnaire were asked to undergo the informed consent process to participate in the study. This included a detailed explanation of the study, the process of randomisation, the possibility of being waitlisted for 2 months, and the required follow-up periods. They received the same explanation in writing, after which they could ask further questions, and were then invited to sign the informed consent.

\section{Interventions}

The counselling approach was based on principles derived from brief trauma-focused therapy, with an 'individual development oriented' approach. ${ }^{19}$ The first task of the counsellor is to make a personal and meaningful contact with the client. ${ }^{20}$ After identifying the main complaint, the counsellor explores any specific precipitating events to gain a clear picture from the client's point of view about the history of the complaint. During subsequent sessions, the counsellor works with the client according to the goals set during the first consultation 


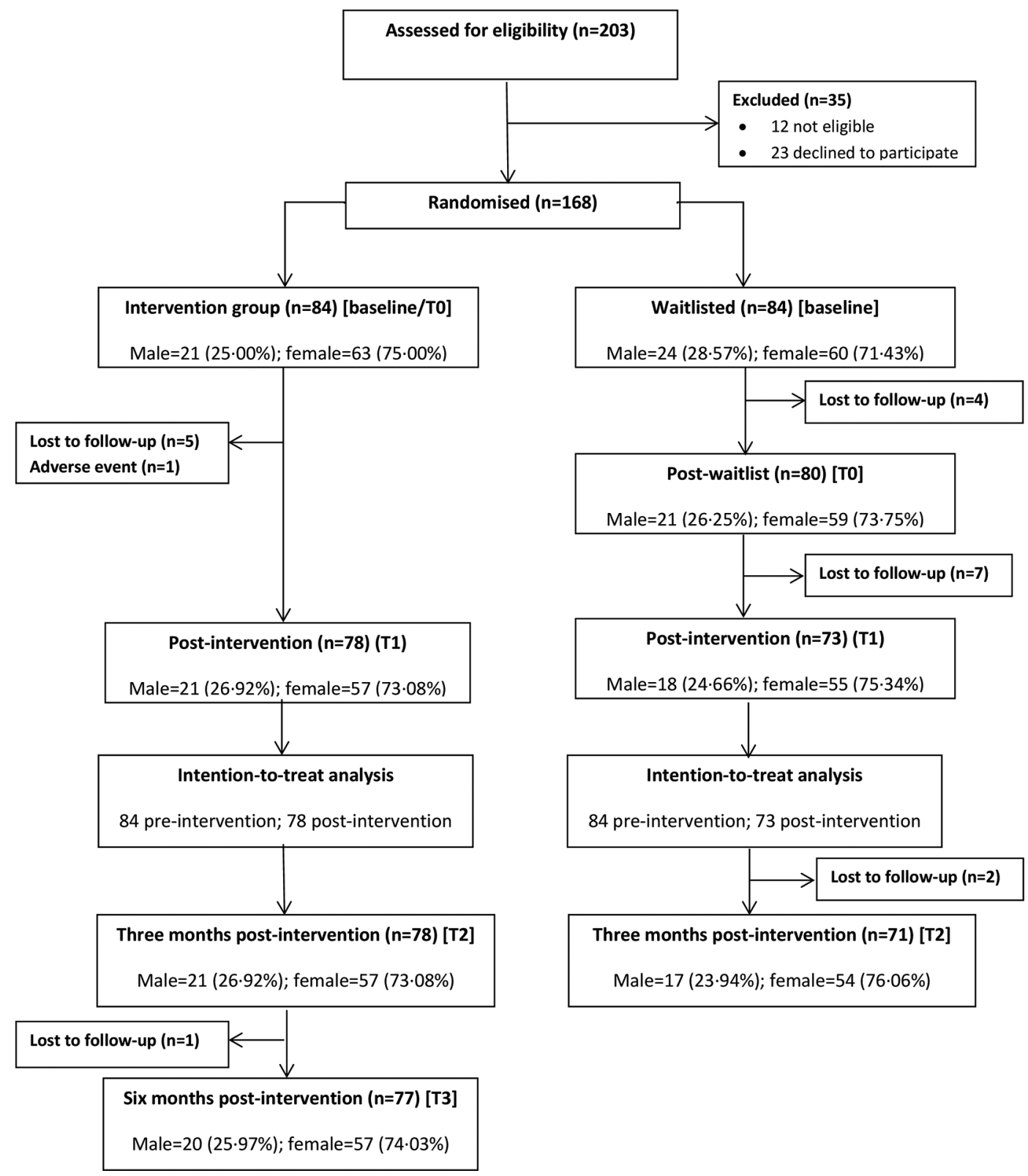

Figure 1 CONSORT flow chart of participants. CONSORT, Consolidated Standards of Reporting Trials. (http://www.consortstatement.org/).

and an individualised treatment plan. No intervention other than individual counselling was offered. Sessions aimed to finish within $50 \mathrm{~min}$, and the number of sessions held was dependent on the needs of the individual, thus there was no formal limit.

The counselling approach was standardised through training modules delivered by trainers from the Netherlands Institute of Psychology. Follow-up supervision and quality control used the MSF-OCA MH guideline, ${ }^{19}$ clinical supervision by international $\mathrm{MH}$ experts based in Grozny and oversight from headquarters-based $\mathrm{MH}$ advisors. The training for the counselling approach included: theory of psychosocial counselling, the five categories of problems that are covered in sessions (lack of practical and social skills, practical problems, inner conflict, overwhelming feelings and trauma-related symptoms), general problems that counsellors might encounter with the clients they see, knowledge/attitudes/skills of the counsellor, intervention options, counselling processes and reporting and referral procedures. Clinical supervision was done on-site by the international $\mathrm{MH}$ experts and through weekly discussions and case reviews. The MH advisor from headquarters would also undertake annual visits in which all aspects of supervision were reviewed. The MH advisor would also have regular telephone/ Skype contact with the MH expert in Grozny to discuss arising questions, issues and progress.

During the study period, there were eight full-time counsellors providing the individual counselling intervention. Most counsellors had an academic background in psychology and had been working for MSF for more than 10 years. Of the eight counsellors, five specialised 
Table 1 Baseline characteristics of participants

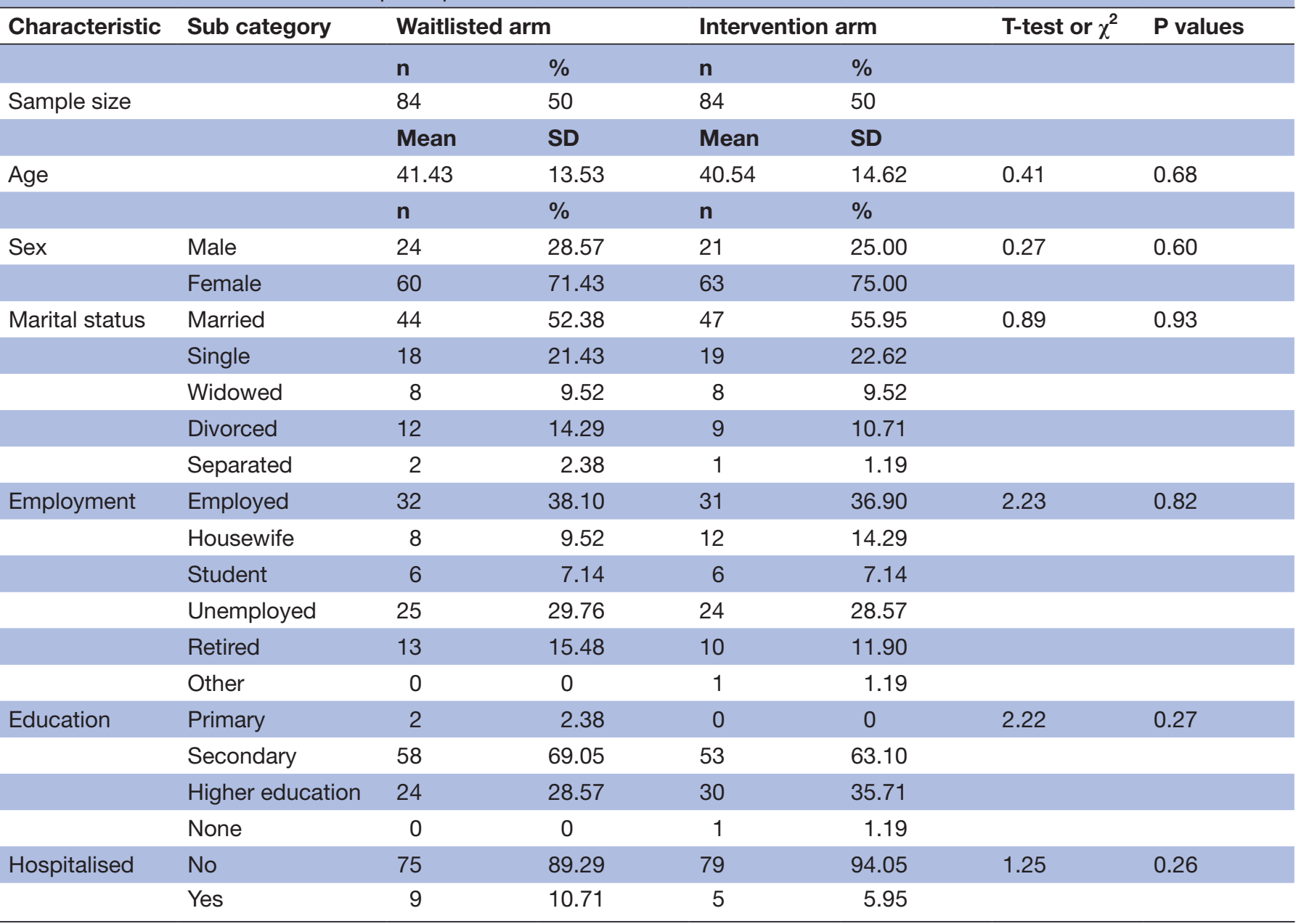

in pedagogy and psychology, one specialised in clinical psychology and two had completed medical school. Six of the eight full-time counsellors were involved in counselling study participants as they were based at the three hospitals, which were the study locations. The remaining two counsellors worked in other locations not included in the study.

\section{Outcomes}

The primary outcome was change in functioning, measured using the Short Form 6 (SF6; an adapted version of the SF36) and locally adapted gender-specific Chechen functioning instruments. ${ }^{21} 22$ The SF6 selected six questions from the SF-36 to assess self-perceived general health, bodily pain, social functioning and role emotional functioning. The SF6 has been used successfully in similar work conducted in war-affected adults in Afghanistan. ${ }^{23}$ The raw scores of each of the four individual items from the SF6 are transformed to fit a 0-100 scale, with high scores representing better functioning. ${ }^{21}$ We also developed and piloted two daily functioning scales for men and women in Chechnya, using an extensive qualitative study before the start of this study. ${ }^{22}$ These gender-specific instruments use a scale from 1 to 4 , including a 'not applicable' score, to rate the participant's abilities to participate in activities considered to be part of daily life for people in Chechnya. The female instrument included 27 items, and the male instrument included 28 items. A reduction in score suggested improvement in functioning.

Secondary outcomes included changes in symptoms of anxiety and depression, coping strategies and perceived social support as measured by the HSCL-25, the Coping Strategy Indicator (CSI) and the Social Provisions Scale (SPS), respectively. ${ }^{24-26}$ We also aimed to measure whether any changes were sustained at 3 and 6 months postintervention. The HSCL-25 evaluates anxiety-related and depression-related symptoms and is rated on a scale from 1 to 4 , where 1 means that the client does not associate with the symptom and 4 means they associate with it 'extremely'. Three scores are calculated: an anxiety score, a depression score and an overall score measuring psychological distress. ${ }^{24}$ The scale was validated in the Russian language in Chechen refugees living in Austria. ${ }^{27}$ Mean overall symptom scores of more than 1.75 for each subcategory have been found to predict clinical diagnosis of anxiety and affective disorders. ${ }^{28} \mathrm{We}$ used this cut-off as an indication of symptoms of mental distress in clients eligible for enrolment in the study. 
Table 2 Average treatment effects $(n=168)$ for functioning: unadjusted scores

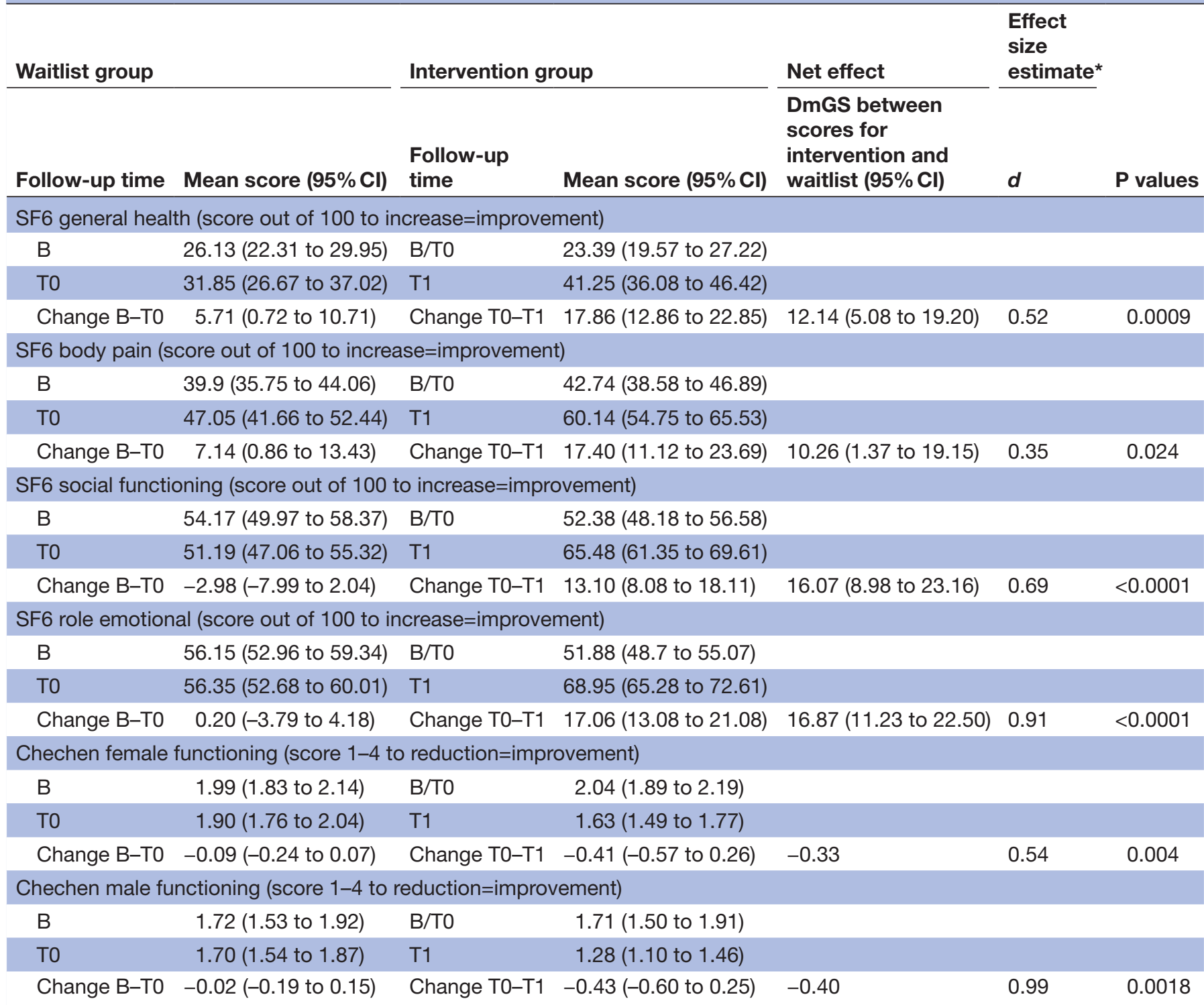

*Measured using Cohen's $d$ statistic.

DmGS, difference between the mean gain scores; SF6, Short Form 6.

The $\mathrm{CSI}^{25}$ includes three subscales of coping strategies: problem solving, avoidance and social support seeking. A reduction in scores indicates an improvement in coping strategies. The 12-item SPS measures perceived social support, ${ }^{26}{ }^{29}$ using a 5-point Likert scale allowing for an unsure response. Participants rated their level of agreement from 1 (strongly disagree) to 5 (strongly agree) with statements regarding respect from others, offering support to others, common interests, feeling supported by others and feeling close to others. An increase in score indicated an improved perception of social support.

Finally, the proportion of study participants with PTSD before and after the intervention was measured using the Harvard Trauma Questionnaire (HTQ-16). ${ }^{30}$ This instrument evaluates the 16 commonly reported symptoms of PTSD on a scale of 1-4, as described in the Diagnostic and Statistical Manual of Mental Disorders, Fourth
Edition (DSM-IV). ${ }^{31}$ It has been used as a screening tool for PTSD in numerous war-affected populations and has been validated in Chechen refugees in Austria. ${ }^{32}$ This definition of PTSD requires a score of 3 or 4 on at least one of four re-experiencing symptoms, at least three of seven avoidance and numbing symptoms and at least two of five arousal symptoms. ${ }^{30}$ Thus, any person that met the aforementioned criteria was considered to be screened positive PTSD. The prevalence of PTSD in the intervention and waitlisted group was calculated by dividing the number of PTSD-screened positive participants by the total number of participants in their study group.

As Chechen is mostly an oral language but the majority of Chechens speak, read and write Russian, we chose to translate the instruments into Russian. All Russian translations were back translated into English to ensure consistency of language. The Russian terminology used in each 
Table 3 Adjusted treatment effects for functioning $(n=168)$

Adjusted DmGS

between intervention

and waitlist groups

(95\% Cl)

\begin{tabular}{llrc} 
& (95\% Cl) & F value & P values \\
\hline $\begin{array}{l}\text { SF6 general } \\
\text { health }\end{array}$ & 11.81 (4.90 to 18.72) & 11.41 & 0.0009 \\
\hline $\begin{array}{l}\text { SF6 body pain } \\
\text { SF6 social } \\
\text { functioning }\end{array}$ & $10.55(1.91$ to 19.19$)$ & 5.82 & 0.017 \\
$\begin{array}{l}\text { SF6 emotional } \\
\text { role }\end{array}$ & 17.05 (11.45 to 22.65$)$ & 36.19 & $<0.0001$ \\
$\begin{array}{l}\text { Chechen female } \\
\text { functioning }\end{array}$ & $-0.36(-0.57$ to 0.14$)$ & 10.79 & 0.0014 \\
$\begin{array}{l}\text { Chechen male } \\
\text { functioning }\end{array}$ & $-0.27(-0.56$ to 0.02$)$ & 3.52 & 0.0691 \\
\hline
\end{tabular}

DmGS, difference between the mean gain scores; SF6, Short Form 6.

instrument was carefully double checked in the piloting process of all instruments. Together with the study interviewers, we created verbal Chechen translations for specific Russian phrases or words that were not well understood by individuals who participated in the pilot of the instruments. As such, study interviewers used the closest term that conveyed the same meaning in Chechen and Russian language for all administered instruments.

\section{Data collection}

A case reporting form (CRF) was created for each participant for each relevant visit. The visits were defined as baseline (B; at enrolment for both groups), T0 (just before the counselling intervention in both groups), T1 (after the intervention for both groups), T2 (3 months after the intervention for both groups) and T3 (6 months after the intervention for the intervention group). This CRF included all study instruments and allowed the study interviewer to collect information on important life events that occurred between visits. Study interviewers were trained for 2 weeks to ensure consistency in language and style of administering the instruments.

Routine data on the counselling intervention was collected separately according to the MSF-OCA MH programme specifications, using a unique patient ID number and no other identifying data. Linkage between the participant's number and their patient ID number could only be done by the study coordinator through a password-protected database.

\section{Sample size}

A sample size of 46 in both the waitlisted and intervention arms was calculated for an estimated effect size (estimated difference in means between intervention and waitlisted divided by common SD) of $0.40,80 \%$ power and an alpha of 0.05 . We conservatively assumed a $45 \%$ loss to follow-up in our total sample, and thus aimed to include 84 participants in each arm for a total sample size of 168 .
Randomisation and masking

Randomisation to the intervention and waitlisted groups used a computer-generated sequence for each hospital. This sequence was only accessible to the study coordinator and study assistant in a password-protected file. The statistician generated the random allocation sequences before the study started, using the Proc Plan procedure in SAS V.9.4.

After participants had provided written informed consent and officially been enrolled, the study interviewers called the study coordinator for information on the allocated study arm of that participant. Participants were informed of their allocated study arm, and a follow-up visit for the counselling intervention or for the follow-up visit after the waitlist period was established. At the next visit (T1 for the intervention group and $\mathrm{T} 0$ for the waitlisted group), the study interviewer was switched to maintain blinding of the allocation arm. Participants were instructed not to reveal their allocation at this visit. Although the study team did their best to ensure blinding up to $\mathrm{T} 1$ for the intervention group and $\mathrm{T} 0$ for the waitlisted group, maintaining this at all times was difficult, as normal conversation between interviewers and participants might have elicited this information. After the postintervention visit, no further blinding was maintained.

\section{Statistical methods}

Descriptive analysis on demographic indicators (sex, age, marital status and so on) for both groups involved calculating means (and SD) for continuous variables and proportions for categorical variables. Differences between the groups were determined using the t-test to compare means and $\chi^{2}$ to test differences between proportions.

For each participant, we calculated individual scores for the SF6, HSCL-25, CSI and SPS at each visit. For the intervention group, we calculated mean changes in individual gain scores between the $\mathrm{B} / \mathrm{T} 0$ and $\mathrm{T} 1$ visits and the mean gain score for this period. For the waitlisted group, we calculated the individual gain scores between enrolment at baseline and T0 after their waitlisted period and the mean gain score for the whole group for the same period. We then compared the difference between the mean gain scores (DmGS) of the groups using mixed regression models accounting for individual change. A multivariable regression model was constructed to estimate the adjusted DmGS between the groups, incorporating the following covariates to adjust for potential confounding: hospital, age, sex, education level, marital status and employment status at enrolment. We also adjusted for the total number of counselling sessions for each participant.

We analysed the data in the intention-to-treat model to test the intervention effects, including all participants enrolled in the study even if they dropped out between the two time points. For participants lost to follow-up, we imputed the last known value from the previous visit.

Cohen's $d$ effect sizes were calculated for the unadjusted differences in gain scores between the groups. We defined 
an effect size of $<0.15$ as negligible, $0.15-0.40$ as small, $0.41-0.75$ as medium and $>0.75$ as large. ${ }^{33}$ The difference between the mean adjusted difference in gains scores for the two groups were compared using the F-statistic and corresponding $\mathrm{p}$ values (equations of the analysis are available in the online supplementary information).

To determine whether the change in scores after the intervention was maintained in both groups, we combined all data for mean scores at T1 and T2 (3 months after finishing counselling). This analysis did not use the intention-to-treat model. We also looked at maintenance of the score in the intervention group 6 months after counselling finished (T3). Mixed regression models were used to measure the difference in the mean of the pooled scores between T1 and T2 (both groups combined) and between T2 and T3 (intervention group only). The analysis was only performed on those participants for whom we had outcome measures at these time points.

For the HTQ-16 (PTSD), we calculated prevalence of PTSD in the two groups at all visits by identifying participants meeting DSM-IV criteria. We compared the change in prevalence in the intervention group between $\mathrm{B} / \mathrm{T} 0$ and $\mathrm{T} 1$ with the change in prevalence in the waitlisted group between B and T0, using logistic regression estimated by generalised estimating equations to account for the repeated measures of PTSD. The model included an interaction term for group and time to account for the change in the PTSD status of participants in each group between the two time points and adjusted for sex, hospital of recruitment and age. Measures of association were calculated as ORs with $95 \%$ CIs and $p$ values. This analysis was not using the intention-to-treat model.

All analysis was done in STATA V.13.0 and SAS V.9.4.

\section{Ethical considerations}

In February 2013, a community consultation about this study was conducted in Grozny with six groups of participants including nurses, social workers, pharmacy staff and patients in the hospital. ${ }^{18}$ The outcomes were used to inform the final study design (notably reducing the waitlist period from 3 months to 2 months). The study protocol was also reviewed at Centers for Disease Control and Prevention (CDC) and determined to meet the criteria for non-engagement of CDC staff in human subjects' research.

Informed consent forms were translated into Russian and verbal Chechen, back-translated into English and piloted in the area of the study. We explained to clients that participation in the study was voluntary, that they could discontinue from the study at any time without explanation and that they would receive the same standard of treatment whether they agreed to participate. Participants were reimbursed approximately US $\$ 10$ to cover transport costs for each study-related follow-up visit.

\section{Patient and public involvement}

Prior to the implementation of this RCT, two community-based studies were carried out in Grozny in preparation for this study. The first was a community consultation that discussed the stepped-wedge design of the RCT and the acceptability around the waitlist group and the length of the waitlisted period. ${ }^{18}$ As mentioned before, the waitlisted period for this RCT was reduced from the proposed 3 months to 2 months following this community consultation. The second study involved the design of culturally adapted instruments to measure mental distress in the current RCT. ${ }^{22}$ We assumed that the communities involved in these two prior studies were the same as those from which the participants of the RCT arose. Also, at the end of the study, all participants were offered an appointment (and transport costs) to review their own results.

\section{RESULTS \\ Participant recruitment and follow-up}

Between 17 November 2014 and 9 February 2015, we assessed 203 patients presenting for care at the MSF MH programme for eligibility. Thirty-five people were not eligible $(n=12)$ or declined to participate $(n=23)$. Those who declined to participate in the study cited the following reasons: personal reasons $(\mathrm{n}=11,47.8 \%)$, unwilling to wait for counselling $(n=6,26.1 \%)$, distance too far for counselling $(n=3,13.0 \%)$, no permission from family to attend counselling $(\mathrm{n}=1)$, lack of time to attend counselling $(\mathrm{n}=1)$ and for one individual this information was missing. We randomised 168 participants: 84 to each group.

One participant's MH condition deteriorated immediately following enrolment into the study (intervention group); the participant was referred for psychiatric care before starting the intervention and was no longer followed in the study. We lost another 19 participants to follow-up (11.30\%): 6 (7.14\%) in the intervention group (five during the intervention and one between 3 months and 6 months postintervention) and $13(15.48 \%)$ in the waitlisted group (four during the waitlisted period, seven during the intervention and two between the end of counselling and 3-month follow-up). The total proportion lost to follow-up did not differ between the groups ( $\mathrm{p}=0.082)$ (figure 1).

\section{Baseline data}

Demographic characteristics of the intervention and waitlisted group participants were similar; most were female, married and employed, had completed high school and were not hospitalised at the time of enrolment (table 1). Out of the 14 hospitalised participants, it should be noted that they were all hospitalised for non-MH-related reasons. There was no evidence of differences in characteristics of the two groups $(\mathrm{p} \geq 0.26)$.

For the 78 intervention group participants who completed counselling, information was available for 76 participants on the number of counselling sessions completed and the duration of those sessions (mean sessions: $3.8 \quad(\mathrm{SD}=0.7)$; duration: 29.7 days $(\mathrm{SD}=9.7))$. Seventy-one waitlisted participants for whom this 
Table 4 Average treatment effects $(n=168)$ for symptoms, coping and perceived social support: unadjusted scores

\begin{tabular}{|c|c|c|c|c|c|c|}
\hline \multicolumn{2}{|l|}{ Waitlist group } & \multicolumn{2}{|c|}{ Intervention group } & \multirow{2}{*}{$\begin{array}{l}\text { Net effect } \\
\text { DmGS between } \\
\text { scores for } \\
\text { intervention and } \\
\text { waitlist }(95 \% \mathrm{Cl})\end{array}$} & $\begin{array}{l}\text { Effect } \\
\text { size } \\
\text { estimate }^{*}\end{array}$ & \multirow[b]{2}{*}{$P$ values } \\
\hline Follow-up time & Mean score $(95 \% \mathrm{Cl})$ & $\begin{array}{l}\text { Follow-up } \\
\text { time }\end{array}$ & Mean score $(95 \% \mathrm{Cl})$ & & d & \\
\hline \multicolumn{7}{|c|}{ HSCL-25 Anxiety (score 1-4 to reduction=improvement) } \\
\hline $\mathrm{B}$ & 2.49 (2.40 to 2.58$)$ & $\mathrm{B} / \mathrm{TO}$ & 2.54 (2.45 to 2.63$)$ & & & \\
\hline TO & 2.21 (2.10 to 2.33$)$ & T1 & $1.71(1.6$ to 1.83$)$ & & & \\
\hline Change B-TO & $-0.28(-0.40$ to 0.17$)$ & Change T0-T1 & $-0.83(-0.94$ to 0.71$)$ & $-0.55(-0.71$ to 0.39$)$ & 1.03 & $<0.0001$ \\
\hline \multicolumn{7}{|c|}{ HSCL-25 Depression (score 1-4 to reduction=improvement) } \\
\hline $\mathrm{B}$ & 2.43 (2.33 to 2.52$)$ & $\mathrm{B} / \mathrm{TO}$ & 2.43 (2.34 to 2.52$)$ & & & \\
\hline TO & 2.23 (2.13 to 2.34$)$ & T1 & $1.74(1.63$ to 1.85$)$ & & & \\
\hline Change B-TO & $-0.19(-0.30$ to 0.09$)$ & Change T0-T1 & $-0.69(-0.79$ to 0.58$)$ & $-0.50(-0.65$ to 0.35$)$ & 1.01 & $<0.0001$ \\
\hline \multicolumn{7}{|c|}{ HSCL-25 Total (score 1-4 to reduction=improvement) } \\
\hline $\mathrm{B}$ & 2.45 (2.37 to 2.54$)$ & $\mathrm{B} / \mathrm{TO}$ & 2.48 (2.39 to 2.56$)$ & & & \\
\hline TO & 2.22 (2.12 to 2.33$)$ & T1 & 1.73 (1.63 to 1.83$)$ & & & \\
\hline Change B-TO & $-0.23(-0.33$ to 0.13$)$ & Change T0-T1 & $-0.74(-0.85$ to 0.65$)$ & $-0.52(-0.66$ to 0.37$)$ & 1.11 & $<0.0001$ \\
\hline \multicolumn{7}{|c|}{ Coping problem solving (score $1-3$ to reduction=improvement) } \\
\hline $\mathrm{B}$ & $1.58(1.50$ to 1.67$)$ & $\mathrm{B} / \mathrm{TO}$ & 1.54 (1.45 to 1.62$)$ & & & \\
\hline TO & $1.58(1.50$ to 1.66$)$ & T1 & $1.64(1.56$ to 1.72$)$ & & & \\
\hline Change B-TO & $-0.01(-0.10$ to 0.09$)$ & Change T0-T1 & 0.10 (0.01 to 0.19$)$ & $0.11(-0.02$ to 0.24$)$ & 0.25 & 0.103 \\
\hline \multicolumn{7}{|c|}{ Coping social support (score $1-3$ to reduction=improvement) } \\
\hline $\mathrm{B}$ & 2.12 (2.01 to 2.23$)$ & $\mathrm{B} / \mathrm{TO}$ & 2.08 (1.97 to 2.19$)$ & & & \\
\hline TO & 2.12 (2.01 to 2.22$)$ & $\mathrm{T} 1$ & 2.06 (1.96 to 2.16$)$ & & & \\
\hline Change B-TO & $0(-0.12$ to 0.12$)$ & Change T0-T1 & $-0.02(-0.14$ to 0.10$)$ & $-0.01(-0.18$ to 0.16$)$ & 0.02 & 0.871 \\
\hline \multicolumn{7}{|c|}{ Coping avoidance (score $1-3$ to reduction=improvement) } \\
\hline $\mathrm{B}$ & $2.13(2.05$ to 2.21$)$ & $\mathrm{B} / \mathrm{TO}$ & $2.10(2.01$ to 2.18$)$ & & & \\
\hline TO & 2.11 (2.02 to 2.20$)$ & T1 & 2.38 (2.30 to 2.47$)$ & & & \\
\hline Change B-TO & $-0.02(-0.07$ to 0.11$)$ & Change T0-T1 & $0.29(-0.20$ to 0.38$)$ & 0.31 (0.18 to 0.44$)$ & 0.72 & $<0.0001$ \\
\hline \multicolumn{7}{|c|}{ Perceived social support (score out of 60 to increase=improvement) } \\
\hline $\mathrm{B}$ & 44.33 (43.34 to 45.33$)$ & B/TO & 44.23 (43.23 to 45.22$)$ & & & \\
\hline TO & 44.96 (44.05 to 45.88$)$ & $\mathrm{T} 1$ & 46.75 (45.84 to 47.67$)$ & & & \\
\hline Change B-TO & $0.63(-0.32$ to 1.58$)$ & Change T0-T1 & 2.52 (1.58 to 3.47 ) & 1.89 (0.55 to 3.23$)$ & 0.43 & 0.006 \\
\hline
\end{tabular}

*Measured using Cohen's $d$ statistic.

DmGS, difference between the mean gain scores; HSCL-25, Hopkins Symptom Checklist-25.

information was available (of the 73 participants who completed counselling) had a higher mean number of counselling sessions $(4.1 \quad(\mathrm{SD}=0.6)$; t-value $=2.1, \mathrm{p}=0.04)$ and mean duration of counselling (33.6 days $(\mathrm{SD}=12.0)$; $\mathrm{t}$-value $=2.2 ; \mathrm{p}=0.03)$. The presenting complaint for counselling as assessed by the counsellor was similar between the groups $\left(\chi^{2}=5.5, \mathrm{p}=0.6\right)$; most sought counselling for anxiety-related $(\mathrm{n}=54,36.7 \%)$, mood-related $(\mathrm{n}=34$, $23.1 \%)$, family-related $(\mathrm{n}=22,15.0 \%)$ and behaviour-related complaints $(n=20,13.6 \%)$. The precipitating event leading to seeking counselling was also similar between groups $\left(\chi^{2}=10.3, p=0.2\right)$, with similar proportions seeking care due to psychological violence $(n=43,39.3 \%)$, domestic discord or domestic violence $(n=41,27.9 \%)$ or non-violence-related events $(n=41,27.9 \%)$. During the intervention, the counselling focus was similar in the two groups $\left(\chi^{2}=1.62, p=0.8\right)$. The counselling focus was on practical problems $(\mathrm{n}=58,39.5 \%)$, overwhelming feelings $(\mathrm{n}=32,21.8 \%)$, trauma-related symptoms $(\mathrm{n}=29,19.7 \%)$, inner problems $(\mathrm{n}=17,11.6 \%)$ and lack of essential practical or social skills $(n=11,7.5 \%)$. (Please note that this data is collected in our routine $\mathrm{MH}$ programme data. For further details on how this information is collected, please contact the corresponding author). 
Table 5 Adjusted treatment effects for symptoms and coping, and social support $(n=168)$

\begin{tabular}{|c|c|c|c|}
\hline & $\begin{array}{l}\text { Adjusted } \\
\text { DmGS between } \\
\text { intervention and } \\
\text { waitlist groups } \\
(95 \% \mathrm{Cl})\end{array}$ & F value & $P$ values \\
\hline \multicolumn{4}{|l|}{ Symptoms } \\
\hline $\begin{array}{l}\text { HSCL } 25 \\
\text { Anxiety }\end{array}$ & $-0.56(-0.72$ to 0.39$)$ & 46.06 & $<0.0001$ \\
\hline $\begin{array}{l}\text { HSCL } 25 \\
\text { Depression }\end{array}$ & $-0.51(-0.65$ to 0.36$)$ & 44.90 & $<0.0001$ \\
\hline $\begin{array}{l}\text { HSCL } 25 \\
\text { Overall }\end{array}$ & $-0.53(-0.67$ to 0.38$)$ & 53.71 & $<0.0001$ \\
\hline \multicolumn{4}{|c|}{ Coping and social support } \\
\hline Problem solving & 0.12 (0.02 to 0.25$)$ & 3.05 & 0.0825 \\
\hline Social support & $-0.02(-0.19$ to 0.16$)$ & 0.03 & 0.8622 \\
\hline Avoidance & 0.30 (0.17 to 0.43$)$ & 20.16 & $<0.0001$ \\
\hline $\begin{array}{l}\text { Perceived } \\
\text { social support }\end{array}$ & 2.03 (0.68 to 3.37 ) & 8.83 & 0.0034 \\
\hline
\end{tabular}

DmGS, difference between the mean gain scores; HSCL-25, Hopkins Symptom Checklist-25.

\section{Numbers analysed}

For the primary outcome and secondary outcomes looking at differences in mean gain scores between the groups from $\mathrm{T} 0$ and $\mathrm{T} 1$ for the intervention group and baseline and T0 for the waitlisted group, we included 84 participants in both groups with imputed data on scores from the last known follow-up visit. For PTSD analysis, we compared the difference in PTSD prevalence in the intervention group between B/T0 (84 participants) and T1 (80 participants) to that in the waitlisted participants between B (84 participants) and T0 (80 participants). For the maintenance of scores after the intervention, we included 78 people in the intervention group and 71 from the waitlisted group.

\section{Functioning}

Using the SF6, the counselling intervention had moderate to large effect sizes in the intervention group compared with the waitlisted group (general health DmGS=12.14, effect size $=0.52$; bodily pain DmGS=10.26, effect size $=0.35$; social functioning DmGS=16.07, effect size=0.69; and role emotional DmGS=16.87, effect size=0.91) (table 2). The Chechen functioning instruments showed a DmGS of -0.33 (effect size $=0.54$ ) for females and a DmGS of -0.40 (effect size $=0.99$ ) for males. In the adjusted analysis, the intervention group also showed improved functioning by SF6 and the Chechen female functioning scale (all $\mathrm{p}<0.05$ ) (table 3). For the male score, the adjusted analysis showed less strong evidence of an improvement in functioning in the intervention group compared with the waitlisted group $(\mathrm{F}=3.52, \mathrm{p}=0.0691)$.

\section{Anxiety and depression}

The unadjusted DmGS between the intervention and waitlisted groups using the HSCL-25 was -0.55 for anxiety symptoms, -0.50 for depression symptoms and -0.52 for overall psychological distress (table 4). The effect sizes calculated in the unadjusted analysis for all three measurements were large (all $d>1.0$ and $\mathrm{p}<0.0001$ ). This positive impact of counselling in the intervention group compared with the waitlisted group was maintained in the adjusted analysis, with $\mathrm{p}<0.0001$ for all three measurements (table 5).

\section{Coping and perceived social support}

Using the CSI, the unadjusted DmGS related to problem solving and social support were very small with small effect sizes (problem solving DmGS=0.11, $d=0.25, \mathrm{p}=0.103$; social support DmGS=-0.01, $d=0.02, \mathrm{p}=0.871$ ) (table 4$)$. In the adjusted analysis, problem solving and social support showed no significant evidence of difference between the groups $(\mathrm{p}=0.08$ and $\mathrm{p}=0.86$, respectively) (table 5). The coping scale for avoidance showed a significant worsening, with a moderate effect size (DmGS $=0.31, d=0.72$, $\mathrm{p}<0.0001)$. In the adjusted analysis, the worsening in avoidance coping skills remained $(\mathrm{F}=20.16, \mathrm{Pp}<0.001)$. Perceived social support improved in the intervention group compared with the waitlisted group, with a moderate effect size (DmGS=1.89, $d=0.43, \mathrm{p}=0.006)$, and this remained relevant in the adjusted analysis $(\mathrm{F}=8.83$, $\mathrm{p}=0.0034)($ table 5).

\section{Maintenance of scores after counselling}

A continued improvement was observed using the scales for SF6 social functioning, SF6 role emotional and the Chechen female and male functioning scales at T2 compared with T1 (all $\mathrm{p}<0.05)$. The effect was maintained at T3 with no further improvement for SF6 social functioning $(p=0.60)$, SF6 role emotional $(p=0.25)$ or the Chechen male functioning scale $(p=0.17)$. Further significant improvement was observed at T3 compared with $\mathrm{T} 2$ in female participants from the intervention group $(p=0.02)$. The effect of counselling was sustained (but not further improved) at T2 compared with T1 when measured on the SF6 general health $(p=0.38)$ and body pain $(p=0.10)$ scales. Similar trends were observed for these two scales at T3 compared with T2 ( $\mathrm{p}=0.94$ and $\mathrm{p}=0.95$, respectively) (table 6 ).

Symptoms of anxiety, depression and psychological distress, measured by the HSCL-25, improved further at $\mathrm{T} 2$ compared with $\mathrm{T} 1(\mathrm{p}=0.03, \mathrm{p}=0.003$ and $\mathrm{p}=0.005$, respectively). This improvement continued in the intervention group at T3 compared with T2 $(\mathrm{p}=0.0009, \mathrm{p}=0.001$ and $\mathrm{p}=0.0005$, respectively). Results for all aspects of the CSI showed either no change or a slight deterioration at T2 and T3. For perceived social support, a small improvement was observed at $\mathrm{T} 2(\mathrm{p}=0.002)$ compared with $\mathrm{T} 1$, which was sustained but not further improved at T3 in the intervention group (table 7). 
Table 6 Sustained improvement for functioning (unadjusted scores)

\section{Postintervention to 3-month follow-up (T1-T2) to $n=149$} (intervention and waitlist groups combined)

\begin{tabular}{|c|c|c|c|c|c|}
\hline Follow-up visit & Mean score $(95 \% \mathrm{Cl})$ & $P$ values & Follow-up visit & Mean score $(95 \% \mathrm{Cl})$ & $P$ values \\
\hline \multicolumn{6}{|c|}{ SF6 general health (score out of 100 , increase=improvement) } \\
\hline T1 & $41.78(37.77$ to 45.78$)$ & & T2 & $41.88(36.30$ to 47.47$)$ & \\
\hline T2 & 39.97 (36.05 to 43.88$)$ & & T3 & 42.08 (36.67 to 47.48$)$ & \\
\hline Pre-post change & $-1.81(-5.88$ to 2.26$)$ & 0.38 & Pre-post change & $0.19(-4.71$ to 5.10$)$ & 0.94 \\
\hline \multicolumn{6}{|c|}{ SF6 body pain (score out of 100 , increase=improvement) } \\
\hline T1 & 60.72 (56.69 to 64.76$)$ & & T2 & $66.96(61.04$ to 72.88 & \\
\hline T2 & 64.40 (60.43 to 68.37$)$ & & T3 & 67.14 (61.26 to 73.03$)$ & \\
\hline Pre-post change & $3.68(-0.70$ to 8.06$)$ & 0.10 & Pre-post change & $0.18(-5.32$ to 5.68$)$ & 0.95 \\
\hline \multicolumn{6}{|c|}{ SF6 social functioning (score out of 100 , increase=improvement) } \\
\hline T1 & 65.27 (61.89 to 68.65$)$ & & T2 & 71.10 (65.89 to 76.32$)$ & \\
\hline T2 & 70.64 (67.25 to 74.02$)$ & & T3 & 72.73 (67.93 to 77.53$)$ & \\
\hline Pre-post change & 5.37 (1.47 to 9.26$)$ & 0.007 & Pre-post change & $1.62(-4.44$ to 7.68$)$ & 0.60 \\
\hline \multicolumn{6}{|c|}{$\begin{array}{l}\text { SF6 role emotional (score out of } 100, \\
\text { increase=improvement) }\end{array}$} \\
\hline T1 & $69.30(66.55$ to 72.04$)$ & & $\mathrm{T} 2$ & 75.00 (70.88 to 79.12$)$ & \\
\hline T2 & 73.88 (70.92 to 76.84$)$ & & T3 & 77.49 (73.60 to 81.38$)$ & \\
\hline Pre-post change & 4.59 (1.36 to 7.81$)$ & 0.006 & Pre-post change & $2.49(-1.76$ to 6.74$)$ & 0.25 \\
\hline \multicolumn{6}{|c|}{$\begin{array}{l}\text { Chechen female functioning ( } \mathrm{n}=111 \text { and } \mathrm{n}=57 \text { ) } \\
\text { (score } 1-4 \text {, reduction=improvement) }\end{array}$} \\
\hline T1 & $1.53(1.44$ to 1.63$)$ & & T2 & $1.42(1.31$ to 1.53$)$ & \\
\hline T2 & $1.42(1.33$ to 1.51$)$ & & T3 & $1.28(1.21$ to 1.36$)$ & \\
\hline Pre-post change & $-0.12(-0.22$ to -0.01$)$ & 0.03 & Pre-post change & $-0.14(-0.25$ to -0.02$)$ & 0.02 \\
\hline \multicolumn{6}{|c|}{ Chechen male functioning ( $n=38$ and $n=20$ ) (score $1-4$, reduction=improvement) } \\
\hline T1 & $1.38(1.26$ to 1.50$)$ & & T2 & 1.19 (1.07 to 1.32$)$ & \\
\hline T2 & 1.24 (1.15 to 1.33$)$ & & T3 & $1.12(1.06$ to 1.19$)$ & \\
\hline Pre-post change & $-0.14(-0.25$ to -0.03$)$ & 0.018 & Pre-post change & $-0.07(-0.18$ to 0.03$)$ & 0.17 \\
\hline
\end{tabular}

SF6, Short Form 6.

\section{Post-traumatic stress disorder}

At baseline, $32(\mathrm{n}=84,38.10 \%)$ participants in the intervention group and $39(\mathrm{n}=84,46.43 \%)$ in the waitlisted group were classified as meeting DSM-IV criteria for PTSD using HTQ-16 $\left(\chi^{2}=1.20, \mathrm{p}=0.274\right)$. At T1, the prevalence in the intervention group had reduced $(n=5 / 78$, $6.41 \%)$. The prevalence of PTSD in the waitlisted group was $38.75 \%$ after the waitlisted period at $\mathrm{T} 0$ and had also reduced at $\mathrm{T} 1(\mathrm{n}=8 / 73,10.96 \%)$. The adjusted reduction in PTSD prevalence in the intervention group between baseline/T0 and T1 was significantly lower than that in the waitlisted group between baseline and T0 (OR 0.11; $95 \%$ CI 0.04 to $0.28, \mathrm{p}<0.001$ ) (table 8 ).

\section{DISCUSSION}

We have shown that individual counselling in conflict-affected adults in Chechnya improved their daily functioning. Both study instruments used to test functioning, including one that was specifically designed for use in

3-6 months follow-up (T2-T3) to $\mathrm{n}=77$ (intervention group only)

2.08 (36.67 to 47.48$)$

$66.96(61.04$ to 72.88

67.14 (61.26 to 73.03$)$

71.10 (65.89 to 76.32$)$

72.73 (67.93 to 77.53$)$

Chechen adults, showed an improvement in the intervention group compared with the waitlisted group. The unadjusted and adjusted analyses for the functioning instruments showed very similar results, suggesting that the improvement in the intervention group compared with the waitlisted group was not importantly influenced by age, sex, marital and employment status, number of counselling sessions or hospital of recruitment. The absence of statistical evidence for a true difference in the Chechen male functioning scale is likely due to the study being underpowered to detect differences among subgroups.

The results are especially encouraging as different instruments to measure functioning recorded similar positive outcomes, strongly suggesting that the counselling is effective in improving this aspect of clients' lives. Such positive outcomes have also been shown in a retrospective review of programmatic data from $18 \mathrm{MSF}$ projects with MH programmes in conflict and postconflict 
Table 7 Change in scores for symptoms, coping and social support at 3 and 6 months follow-up (unadjusted scores)

Postintervention to 3-month follow-up (T1-T2) to $\mathrm{n}=149$ (intervention and waitlist groups combined)

Follow-up visit Mean score $(95 \% \mathrm{Cl})$

HSCL-25 Anxiety (score $1-4$, reduction = improvement)

\begin{tabular}{|c|c|c|c|c|c|}
\hline $\mathrm{T} 1$ & 1.63 (1.56 to 1.71$)$ & & $\mathrm{T} 2$ & 1.58 (1.47 to 1.68$)$ & \\
\hline T2 & 1.54 (1.47 to 1.61$)$ & & T3 & 1.39 (1.30 to 1.48$)$ & \\
\hline Pre-post change & $-0.09(-0.18$ to -0.01$)$ & 0.03 & Pre-post change & $-0.19(-0.29$ to -0.08$)$ & 0.0009 \\
\hline
\end{tabular}

HSCL-25 Depression (score 1 - 4, reduction = improvement)

\begin{tabular}{|c|c|c|c|c|c|}
\hline T1 & 1.69 (1.62 to 1.76$)$ & & $\mathrm{T} 2$ & 1.60 (1.50 to 1.69$)$ & \\
\hline T2 & 1.57 (1.51 to 1.64$)$ & & T3 & $1.44(1.36$ to 1.51$)$ & \\
\hline Pre-post change & $-0.12(-0.20$ to -0.04$)$ & 0.003 & Pre-post change & $-0.16(-0.26$ to -0.06$)$ & 0.001 \\
\hline
\end{tabular}

HSCL-25 Total (score $1-4$, reduction = improvement)

\begin{tabular}{|c|c|c|c|c|c|}
\hline T1 & 1.67 (1.60 to 1.74$)$ & & T2 & 1.59 (1.50 to 1.68$)$ & \\
\hline T2 & 1.56 (1.50 to 1.62$)$ & & T3 & 1.42 (1.34 to 1.49$)$ & \\
\hline Pre-post change & $-0.11(-0.19$ to -0.03$)$ & 0.005 & Pre-post change & $-0.17(-0.27$ to -0.08$)$ & 0.0005 \\
\hline
\end{tabular}

Coping problem solving (score $1-3$, reduction = improvement)

\begin{tabular}{|c|c|c|c|c|c|}
\hline $\mathrm{T} 1$ & 1.64 (1.58 to 1.69$)$ & & $\mathrm{T} 2$ & 1.76 (1.65 to 1.86$)$ & \\
\hline T2 & 1.76 (1.69 to 1.82$)$ & & T3 & 1.82 (1.71 to 1.92$)$ & \\
\hline Pre-post change & $0.12(0.05$ to 0.19$)$ & $<0.0001$ & Pre-post change & $0.06(-0.05$ to 0.16$)$ & 0.27 \\
\hline
\end{tabular}

Coping social support (score $1-3$, reduction $=$ improvement)

\begin{tabular}{|c|c|c|c|c|c|}
\hline $\mathrm{T} 1$ & 2.08 (2.01 to 2.15$)$ & & $\mathrm{T} 2$ & 2.12 (2.00 to 2.24$)$ & \\
\hline $\mathrm{T} 2$ & 2.13 (2.05 to 2.20$)$ & & T3 & 2.21 (2.10 to 2.33$)$ & \\
\hline Pre-post change & $0.04(-0.05$ to 1.14$)$ & 0.33 & Pre-post change & $0.09(-0.04$ to 0.22$)$ & 0.18 \\
\hline $\mathrm{T} 1$ & 2.39 (2.33 to 2.44$)$ & & $\mathrm{T} 2$ & 2.46 (2.38 to 2.53$)$ & \\
\hline $\mathrm{T} 2$ & 2.46 (2.41 to 2.51$)$ & & T3 & 2.49 (2.41 to 2.57 ) & \\
\hline \multicolumn{6}{|c|}{ Perceived social support (score out of 60 , increase = improvement) } \\
\hline $\mathrm{T} 1$ & 46.74 (46.12 to 47.36$)$ & & $\mathrm{T} 2$ & 47.88 (47.17 to 48.60$)$ & \\
\hline T2 & 47.75 (47.24 to 48.26$)$ & & T3 & 48.12 (47.40 to 48.84$)$ & \\
\hline Pre-post change & 1.01 (0.37 to 1.66$)$ & 0.002 & Pre-post change & $0.23(-0.60$ to 1.07$)$ & 0.58 \\
\hline
\end{tabular}

HSCL-25, Hopkins Symptom Checklist-25.

Table 8 Unadjusted and adjusted ORs of comparison between change in PTSD prevalence of intervention group preintervention and postintervention compared with waitlisted group

\begin{tabular}{|c|c|c|c|c|}
\hline PTSD & Unadjusted OR (95\% Cl) & P values & Adjusted OR $(95 \% \mathrm{Cl})$ & $P$ values \\
\hline \multicolumn{5}{|l|}{ Waitlist group } \\
\hline$B(n=84)$ & 1.00 & & 1.00 & \\
\hline \multicolumn{5}{|c|}{ Intervention group } \\
\hline B/TO (n=84) & 1.00 & & 1.00 & \\
\hline \multicolumn{5}{|c|}{ Intervention group versus waitlist group } \\
\hline T1 versus $\mathrm{B} / \mathrm{TO}$ & 0.15 (0.05 to 0.45$)$ & 0.001 & $0.14(0.05$ to 0.43$)$ & $<0.001$ \\
\hline
\end{tabular}

PTSD, post-traumatic stress disorder. 
settings. ${ }^{15}$ As this study was a stepped-wedge RCT using validated outcome measures, we provide further evidence that the brief counselling intervention used among people affected by conflict can have a positive impact on their ability to function.

Different counselling strategies in other studies have also shown positive effects on functioning, PTSD, depression and anxiety. Moderate to high improvements in the intervention group compared with the waitlisted group on these outcomes were measured using CETA in Burmese refugees in Thailand and in Iraqi Kurdistan. ${ }^{12} 14$ In contrast, mixed results from group problem-solving counselling in terms of functioning were observed in war-affected adults in Aceh, Indonesia. ${ }^{13}$ A recent RCT in adults with psychological distress in a conflict area in Pakistan identified a large improvement in functioning and symptoms of PTSD, depression and anxiety following 5 weekly sessions of individual counselling delivered by trained lay workers using the WHO $\mathrm{PM}+$ approach. ${ }^{10}$ This study mirrors the intervention described in our study in that it limited the number of counselling sessions to five and did not rely on clinically trained MH professionals. It slightly differs from our approach in that the PM+ is a manualised intervention, whereas the MSF one is a semistructured approach allowing for individual adaptation by the counsellor.

The coping scale did not show evidence for improvement with the intervention, and avoidance appeared to worsen. This suggests that the improvement in functioning and symptoms was not achieved through improvement in the three coping skills measured by the CSI. Alternately, the CSI may not be well adapted to measure coping strategies in this population. The study by our colleagues in $\mathrm{Aceh}^{13}$ also observed a small decline in the use of coping strategies among women in the intervention group.

The SPS showed a significant increase in perceived social support in the intervention group compared with the waitlisted group, which was sustained after completion of counselling. The MSF-OCA MH programme in Grozny includes encouragement to establish positive coping mechanisms (including social support). Social support has been shown to have a positive effect on mental and physical health ${ }^{34}$ and is associated with lower post-traumatic stress scores. ${ }^{35}$ It is also notable that social functioning improved with the intervention.

We were able to determine that there was longer term efficacy of the individual counselling on functioning and symptoms of depression and anxiety in both the intervention and waitlisted groups. Scores on the instruments that tested these items either maintained the improvement or continued to improve at 3 and 6 months after completion of counselling. Studies on Rwandan and Somali refugees in Uganda showed that Narrative Exposure Therapy counselling sustained the reduction in PTSD in all participants at 6 and 12 months' follow-up, although the dropout rates were high. ${ }^{36}$ Very few studies have measured the maintenance of these scores, and thus the current study provides much needed evidence in this regard.

The expression of MH difficulties as physical symptoms, called somatisation, is common in many cultures. ${ }^{37}$ In our study, the functioning scales addressing general health and body pain were not sustained or further improved after completion of counselling. Whether this is due to continuing physical complaints in the study population or because of ongoing psychosomatic complaints, we cannot say. However, we believe the latter is unlikely as the counselling intervention is addressing medically unexplained physical symptoms but did not change or address any physical illness. Neither coping mechanisms nor social support showed any further changes following the end of counselling.

The study has some limitations. Although few of the participants were hospitalised at enrolment, the MSF MH programme is located in hospital settings, which might have biased care-seeking behaviour. The study population therefore might not be entirely representative of those seeking help for $\mathrm{MH}$ concerns in Grozny. We worked hard to ensure that study interviewers remained blinded to the group allocation up to $\mathrm{T} 1$ of the intervention group, but this could not be ensured in all cases. We do not think that this potential bias would have changed our findings substantially, as all instruments had a fixed script for questions to be asked by interviewers and answers provided by participants, not allowing much space for interviewers to influence participants' answers. Grozny represents a specific and long-term unstable situation, so we cannot be certain that similar outcomes would be found in other acute emergencies. The counsellors delivering the intervention, while non-specialised, all had academic training and many years of experience delivering the intervention, which is not the case in many humanitarian settings. However, we have shown previously that although lay counsellors used more sessions than academically trained counsellors, the outcomes in clients were similar. ${ }^{15}$ Also, we conducted a sensitivity analysis that included the counsellor as a covariate in the multivariate regression models, and the results did not change the interpretation of the study findings. This suggests that the positive outcomes measured were not linked to individual counsellors. Finally, we employed widely used instruments to measure MH distress and functioning in this study and thus have equated numerical improvements in the scores of study participants with clinical improvements. This is supported by the knowledge that individual counselling in Grozny was only finalised when the client and counsellor agreed that the main complaint for attending counselling had been resolved (thus improved).

Despite its limitations, this study represents a scientifically rigorous evaluation of an individual counselling intervention in a humanitarian setting, in that it includes a randomised waitlisted group to avoid overestimating improvements due to the effect of time alone. 
We were also able to show that the improvements due to the intervention were maintained and continued to improve at 3 and 6 months after completion of the intervention. This approach both improved functioning in daily life and reduced symptoms of mental distress including anxiety and depression, while decreasing the prevalence of PTSD in adults in Chechnya with similar effect sizes to other interventions. In parallel with the PM+ guidelines, we have shown that low-intensity $\mathrm{MH}$ interventions in humanitarian settings play an important role in improving functioning and reducing psychosocial distress. Further research is needed to determine whether similar results can be shown in acute conflict settings and where less experienced/ trained counsellors deliver the intervention. Additionally, further exploration on the impact of individual counselling in men specifically would be important as they are frequently under-represented in studies in this area.

\section{REGISTRATION}

The study was registered with the Netherlands Trial Register (http://www.trialregister.nl/trialreg/index. asp), which is recognised by the WHO and the International Committee of Medical Journal Editors (ICJME) under registration number NTR4689 (http:/ / www.trialregister.nl/trialreg/admin/rctview.asp?TC=4689). The protocol is available from http://fieldresearch.msf. org/msf/bitstream/10144/618741/1/1326_MSFH+ $\mathrm{MH}+$ Chechnya+outcomes+evaluation+ protocol_ amendment+November+2016_clean_final.pdf.

Acknowledgements We would like to extend our thanks to all the counsellors of the MSF Mental Health Programme in Grozny for their continued dedication and support to the population of Chechnya in confronting and alleviating mental health problems. We would like to thank the Directors of Hospital 7, Hospital 9 and the Regional Hospital in Grozny for allowing this study to take place in these locations. We would like to thank Marise Denault for critical review of the manuscript. Clare Griffith provided editorial assistance to this manuscript. Finally, we would like to thank all study participants for their contribution to this study.

Contributors BL-C, LS, CB, KI, PAB and GP designed the study. CF, ZT, GP and $\mathrm{AL}$ acquired the data. $\mathrm{CF}, \mathrm{CB}$ and $\mathrm{AL}$ analysed the data. All authors contributed to the interpretation of data. AL, BL-C and GP wrote the manuscript, and all authors critically reviewed the manuscript.

Funding All research was carried out by MSF staff as part of their roles.

Disclaimer The findings and conclusions in this report are those of the authors and do not represent the official position of the Centers for Disease Control and Prevention. The corresponding author had full access to all the data in the study and is the final responsible.

Competing interests None declared.

Patient consent Not required.

Ethics approval The study protocol was reviewed and approved by the MSF Ethical Review Board and the Ethical Review Board in Chechnya State University. The study protocol was also reviewed at CDC and determined to meet the criteria for non-engagement of CDC staff inhuman subjects' research.

Provenance and peer review Not commissioned; externally peer reviewed.

Data sharing statement MSF has a managed access system for data sharing. Data are available on request in accordance with MSF's data sharing policy. Requests for access to data should be made to data.sharing@msf.org.
Open access This is an open access article distributed in accordance with the Creative Commons Attribution Non Commercial (CC BY-NC 4.0) license, which permits others to distribute, remix, adapt, build upon this work non-commercially, and license their derivative works on different terms, provided the original work is properly cited, appropriate credit is given, any changes made indicated, and the use is non-commercial. See: http://creativecommons.org/licenses/by-nc/4.0/.

\section{REFERENCES}

1. World Health Organization. mhGAP Intervention Guide - Version 2.0. 2016. http://www.who.int/mental_health/mhgap/mhGAP_ intervention guide $02 / \mathrm{en} /$

2. World Health Organisation (WHO). Problem Management Plus $(\mathrm{PM}+)$ : Individual psychological help for adults impaired by distress in communities exposed to adversity. 2016. http://apps.who.int/iris/ bitstream/10665/206417/1/WHO_MSD_MER_16.2_eng.pdf?ua=1

3. Tol WA, Patel V, Tomlinson M, et al. Research priorities for mental health and psychosocial support in humanitarian settings. PLoS Med 2011;8:e1001096.

4. Blanchet K, Sistenich V, Ramesh A, et al. An evidence review of research on health interventions in humanitarian crises. 2015. http:// www.elrha.org/wp-content/uploads/2015/01/Evidence-Review-22. 10.15.pdf

5. Knaevelsrud C, Brand J, Lange A, et al. Web-based psychotherapy for posttraumatic stress disorder in war-traumatized Arab patients: randomized controlled trial. J Med Internet Res 2015;17:e71.

6. Weiss WM, Murray LK, Zangana GAS, et al. Community-based mental health treatments for survivors of torture and militant attacks in Southern Iraq: a randomized control trial. BMC Psychiatry 2015;15:249.

7. Bolton P, Bass JK, Zangana GA, et al. A randomized controlled trial of mental health interventions for survivors of systematic violence in Kurdistan, Northern Iraq. BMC Psychiatry 2014;14:360.

8. Tol WA, Komproe IH, Jordans MJ, et al. Brief multi-disciplinary treatment for torture survivors in Nepal: a naturalistic comparative study. Int J Soc Psychiatry 2009;55:39-56.

9. Ayoughi S, Missmahl I, Weierstall R, et al. Provision of mental health services in resource-poor settings: a randomised trial comparing counselling with routine medical treatment in North Afghanistan (Mazar-e-Sharif). BMC Psychiatry 2012;12:14.

10. Chiumento A, Hamdani SU, Khan MN, et al. Evaluating effectiveness and cost-effectiveness of a group psychological intervention using cognitive behavioural strategies for women with common mental disorders in conflict-affected rural Pakistan: study protocol for a randomised controlled trial. Trials 2017;18:190.

11. Bass J, Murray SM, Mohammed TA, et al. A randomized controlled trial of a trauma-informed support, skills, and psychoeducation intervention for survivors of torture and related Trauma in Kurdistan, Northern Iraq. Glob Health Sci Pract 2016;4:452-66.

12. Weiss WM, Murray LK, Zangana GA, et al. Community-based mental health treatments for survivors of torture and militant attacks in Southern Iraq: a randomized control trial. BMC Psychiatry 2015;15:249.

13. Bass J, Poudyal B, Tol W, et al. A controlled trial of problem-solving counseling for war-affected adults in Aceh, Indonesia. Soc Psychiatry Psychiatr Epidemiol 2012;47:279-91.

14. Bolton P, Lee C, Haroz EE, et al. A transdiagnostic community-based mental health treatment for comorbid disorders: development and outcomes of a randomized controlled trial among Burmese refugees in Thailand. PLoS Med 2014;11:e1001757.

15. Shanks L, Ariti C, Siddiqui MR, et al. Counselling in humanitarian settings: a retrospective analysis of 18 individual-focused nonspecialised counselling programmes. Confl Health 2013;7:19.

16. Foa EB, Hearst-Ikeda D, Perry KJ. Evaluation of a brief cognitivebehavioral program for the prevention of chronic PTSD in recent assault victims. J Consult Clin Psychol 1995;63:948-55.

17. Brown CA, Lilford RJ. The stepped wedge trial design: a systematic review. BMC Med Res Methodol 2006;6:54.

18. Shanks L, Moroni C, Rivera IC, et al. 'Losing the tombola': a case study describing the use of community consultation in designing the study protocol for a randomised controlled trial of a mental health intervention in two conflict-affected regions. BMC Med Ethics 2015;16:38.

19. De Jong K. Psychosocial and mental health interventions in areas of mass violence: a community based approach. 2nd edn: Médécins Sans Frontières, 2011.

20. Van der Veer G. Training counsellors in areas of armed conflict within a community approach. Utrecht, The Netherlands: Pharos, 2001. 
21. Ware JE, Snow KK, Kosinski M, et al. SF-36 health survey: manual and interpretation guide. 2nd edn. Boston, Massachusetts: The Health Institute, New England Medical Center, 1997.

22. Nguyen AJ, Feo C, Idrisov K, et al. Mental health problems among conflict-affected adults in Grozny, Chechnya: a qualitative study. Confl Health 2016;10:16.

23. Cardozo BL, Bilukha OO, Crawford CA, et al. Mental health social functioning, and disability in postwar Afghanistan. JAMA 2004;292:575-84.

24. Derogatis LR, Lipman RS, Rickels K, et al. The Hopkins Symptom Checklist (HSCL). A measure of primary symptom dimensions. Mod Probl Pharmacopsychiatry 1974;7:79-110.

25. Amirkhan $\mathrm{JH}$. Criterion validity of a coping measure. J Pers Assess 1994;62:242-61.

26. Cutrona CE, Russel D. The provisions of social relationships and adaptation to stress. Adv Pers Relationships 1987;1:37-67.

27. Renner W, Salem I. Post-traumatic stress in asylum seekers and refugees from Chechnya, Afghanistan, and West Africa: gender differences in symptomatology and coping. Int $J$ Soc Psychiatry 2009;55:99-108.

28. Ventevogel P, De Vries G, Scholte WF, et al. Properties of the Hopkins Symptom Checklist-25 (HSCL-25) and the Self-Reporting Questionnaire (SRQ-20) as screening instruments used in primary care in Afghanistan. Soc Psychiatry Psychiatr Epidemiol 2007;42:328-35.

29. Cutrona CE. Ratings of social support by adolescents and adult informants: degree of correspondence and prediction of depressive symptoms. J Pers Soc Psychol 1989;57:723-30.
30. Mollica RF, McDonald L, Massagli M, et al. Measuring trauma, measuring torture: instructions and guidance on the utilization of the harvard program in refugee trauma's versions of the hopkins symptom checklist-25 and the Harvard trauma questionnaire (HTQ). Cambridge, MA, USA, 2004

31. American Psychiatric Association. Diagnostic and statistical. Manual of mental disorders. 4th edn. Arlington, VA, USA: American Psychiatric Association, 2000.

32. Renner W, Salem I, Ottomeyer K. Cross-cultural validation of measures of traumatic symptoms in groups of asylum seekers from Chechnya, Afghanistan, and West Africa. Soc Behav Pers 2006;34:1101-14.

33. Cohen J. Statistical power analysis for the behavioural sciences. 2nd edn. New York: Lawrence Erlbaum Associates, 1988.

34. Ganster DC, Victor B. The impact of social support on mental and physical health. Br J Med Psychol 1988;61:17-36.

35. Ahern J, Galea S, Fernandez WG, et al. Gender, social support, and posttraumatic stress in postwar Kosovo. J Nerv Ment Dis 2004;192:762-70.

36. Neuner F, Onyut PL, Ertl V, et al. Treatment of posttraumatic stress disorder by trained lay counselors in an African refugee settlement: a randomized controlled trial. J Consult Clin Psychol 2008;76:686-94.

37. Gureje O, Simon GE, Ustun TB, et al. Somatization in cross-cultural perspective: a World Health Organization study in primary care. Am J Psychiatry 1997;154:989-95. 